\title{
Criminologie
}

\section{Pour comprendre la pratique du « squeegee » à Montréal}

\section{Véronique Denis}

Volume 36, numéro 2, automne 2003

Le construit de l'ethnicité en criminologie

URI : https://id.erudit.org/iderudit/007868ar

DOI : https://doi.org/10.7202/007868ar

Aller au sommaire du numéro

Éditeur(s)

Les Presses de l'Université de Montréal

ISSN

0316-0041 (imprimé)

1492-1367 (numérique)

Découvrir la revue

Citer cet article

Denis, V. (2003). Pour comprendre la pratique du « squeegee » à Montréal. Criminologie, 36(2), 89-104. https://doi.org/10.7202/007868ar

\section{Résumé de l'article}

La pratique du " squeegee », relativement récente à Montréal et rendue illégale, a été l'objet d'une importante couverture médiatique mais n'a que très peu été étudiée. Même si les adeptes du " squeegee ", communément appelés les "squeegees ", sont souvent considérés comme des " jeunes de la rue ", cet article met en évidence l'hétérogénéité de leurs expériences de vie ainsi que de leur rapport à cette pratique, rendant réductrice cette catégorisation. En outre, la méthodologie qualitative, incluant des observations sur le terrain et des entretiens non directifs auprès de jeunes qui pratiquaient le « squeegee » en 1999, permet d'esquisser le contexte social entourant la pratique du " squeegee " à Montréal. Enfin, à partir des sens que cette pratique revêt pour les jeunes qui l'exercent, l'article examine en quoi la pratique du "squeegee ", socialement vue comme " hors normes ", pourrait s'avérer pour eux un agent d'insertion sociale marginalisée.
Ce document est protégé par la loi sur le droit d'auteur. L'utilisation des services d’Érudit (y compris la reproduction) est assujettie à sa politique d'utilisation que vous pouvez consulter en ligne.

https://apropos.erudit.org/fr/usagers/politique-dutilisation/ 


\title{
Pour comprendre la pratique du « squeegee » à Montréal ${ }^{1}$
}

\author{
Véronique Denis \\ Candidate au doctorat \\ École de criminologie \\ Université de Montréal. \\ denis_veronique@hotmail.com
}

RÉSUMÉ • La pratique du «squeegee ${ }^{2}$, relativement récente à Montréal et rendue illégale, a été l'objet d'une importante couverture médiatique mais n'a que très peu été étudiée. Même si les adeptes du «squeegee», communément appelés les «squeegees», sont souvent considérés comme des «jeunes de la rue», cet article met en évidence l'hétérogénéité de leurs expériences de vie ainsi que de leur rapport à cette pratique, rendant réductrice cette catégorisation. En outre, la méthodologie qualitative, incluant des observations sur le terrain et des entretiens non directifs auprès de jeunes qui pratiquaient le «squeegee» en 1999, permet d'esquisser le contexte social entourant la pratique du «squeegee» à Montréal. Enfin, à partir des sens que cette pratique revêt pour les jeunes qui l'exercent, l'article examine en quoi la pratique du «squeegee», socialement vue comme «hors normes», pourrait s'avérer pour eux un agent d'insertion sociale marginalisée.

ABSTRACT • Squeegeeing is a relatively new and illegal practice in Montreal. The media has talked a lot about this practice but there have been few proper studies of the phenomenon. This article shows that, while the young people that adopt this practice are generally regarded as «squeegee» and as "street youth», the variety of their experiences and motives belies any such reductive categorizations. By way of qualitative methodology, including in-depth interviews and field observations carried out in 1999, the social context of squeegeeing is illustrated. Finally, starting from the meaning that squeegeeing has for those who practice it, the author examines how this activity, despite being viewed as an «outlaw» practice, may allow these young people a degree of social integration, however marginalized.

1. Cet article s'inspire du mémoire de maîtrise de l'auteure, La pratique du squeegee à Montréal : expériences et perceptions des jeunes impliqués, mémoire déposé à l'École de criminologie en 2001. L'auteure remercie chaleureusement Mesdames Marie-Andrée Bertrand, Lise Harou et Geneviève Denis pour leur lecture critique des différentes versions de cet article.

2. Activité (pratiquée par des jeunes) qui consiste à laver, contre rémunération, le pare-brise des voitures à l'arrêt des automobilistes aux intersections. 


\section{Introduction}

Apparue à Montréal au milieu des années 1990, la pratique du «squeegee» a suscité un engouement parmi les jeunes dits «de la rue», d'où son expansion au sein de la métropole. Cette pratique, qui consiste à nettoyer le pare-brise des voitures immobilisées à un feu rouge en échange d'une contribution financière, a donné lieu à une réaction sociale diversifiée allant de la sympathie à la réprobation jusqu'à la répression.

Graduellement perçue comme socialement dérangeante, la pratique $\mathrm{du}$ «squeegee» a engendré plusieurs discussions de nature politique visant à l'encadrer et à la contrôler, ce qui a mené à sa criminalisation en 1996. Cette pratique, et spécialement les jeunes qui l'exercent, a aussi été l'objet d'une curiosité. En regard du traitement médiatique qui leur a été réservé, les «squeegees» sont représentés comme des punks à la chevelure flamboyante, à la tenue délabrée, au tempérament contestataire et aventureux; ils sont pour ainsi dire devenus le symbole d'une marginalité nouvelle à Montréal (Courville, 1998). Un journaliste les a même proclamés «Personnalité de l'année 1997 » en vertu de leur entrepreneurship et de leur débrouillardise (voir Grenier, 1997). D’autres en ont toutefois dressé un portrait plutôt négatif, réducteur et sensationnaliste en focalisant sur leur consommation de drogues et sur leur mode de vie «décadent» (voir Pigeon, 1999). Or, derrière ces représentations médiatiques, aussi variées soient-elles, se cache une réalité plus complexe. En outre, malgré son importante médiatisation, la pratique du «squeegee»a été peu étudiée ${ }^{3}$ et les jeunes qui l'exercent se sont peu exprimés sur ce sujet ${ }^{4}$.

C'est pourquoi il a paru opportun de réaliser une étude qualitative donnant la parole aux adeptes du «squeegee» à Montréal, afin de mieux comprendre leur pratique à travers le sens qu'elle revêt pour eux et la place qu'elle prend dans leur vie. Le présent article en fait partiellement état. Il s'agira d'abord de définir les jeunes adeptes du «squeegee» de manière à tenir compte de la diversité de leurs expériences de vie, de même que de leur rapport à la rue et à la pratique du «squeegee». Par la suite, après

3. Seuls trois documents ont spécifiquement traité de la pratique du «squeegee», soit pour en dresser un portrait général à l'aide d'une recension des écrits (Courville, 1998), soit pour faire le point sur son encadrement judiciaire (Crago, 1998) ou encore pour évaluer la situation en vue de soumettre des recommandations à la Ville de Montréal (Foisy, 1999).

4. Un documentaire intitulé S.P.I.T : Squeegee Punks in Traffic, réalisé en 2001 par Daniel Cross, a été presque entièrement tourné par Roach, un jeune montréalais qui vit dans la rue et qui pratique le «squeegee». Ce film plonge dans son univers et «porte la voix des jeunes de la rue». Pour plus de détails sur ce documentaire, consulter le site internet www.spit.ca. 
avoir dépeint le contexte social entourant la pratique du «squeegee» à Montréal, il s'agira de mettre en lumière l'insertion sociale marginalisée qu'elle favorise chez ceux qui l'ont adoptée à partir du sens qu'elle revêt pour eux. Avant toute chose, exposons brièvement la démarche qui a soutenu la réalisation de cette recherche.

\section{Démarche de recherche}

Dans le cadre de cette recherche, une place centrale a été accordée au discours des adeptes du «squeegee» et à leur subjectivité. Cette démarche rejoint l'une des prémisses de l'approche qualitative selon laquelle «les conduites sociales ne pourraient être comprises ni expliquées en dehors de la perspective des acteurs sociaux» (Poupart, 1997 : 175). C'est donc par l'entremise d'une méthodologie qualitative et, plus spécifiquement, d'observations sur le terrain et d'entretiens non directifs, que les données ont été recueillies.

Les observations sur le terrain se sont déroulées durant l'été et l'automne 1999 dans les rues du centre-ville de Montréal et dans un organisme communautaire qui offre des services aux jeunes de la rue. Elles ont été un moyen privilégié pour prendre contact avec des jeunes qui pratiquaient le «squeegee», pour développer avec eux des liens de confiance et pour saisir le contexte dans lequel le «squeegee» se pratiquait. De la trentaine d'adeptes $\mathrm{du}$ «squeegee» rencontrés lors des observations, quinze se sont prêtés à un entretien qualitatif. L'échantillon, constitué de façon à représenter des expériences les plus diversifiées possible, comprenait douze jeunes hommes et trois jeunes femmes, dont l'âge variait de moins de dixhuit ans à vingt-sept ans. Ceux-ci pratiquaient le «squeegee» depuis une période allant d'une journée à cinq ans. Si la grande majorité des répondants ne possédaient pas de diplôme d'études secondaires, cinq d'entre eux avaient réintégré le milieu scolaire lors des entretiens. Neuf répondants habitaient en chambre ou en appartement et recevaient des prestations de la sécurité du revenu. Enfin, neuf des quinze répondants consommaient, ou avaient déjà consommé, des drogues injectables.

\section{Au-delà de la pratique du "squeegee »: des jeunes en situation de rue}

Les «squeegees», tel qu'ils sont socialement désignés, ne forment pas un groupe social homogène et distinct, pas plus qu'ils ne sont tous des jeunes 
de la rue. En effet, cette catégorie «jeunes de la rue», bien qu'elle ne bénéficie pas d'une définition consensuelle parmi les chercheurs (Brannigan et Caputo, 1993; Laberge et Roy, 1994; Bellot, 2001), tend à être définie par un sentiment d'appartenance que les jeunes développeraient envers le milieu de la rue, c'est-à-dire envers des lieux et des activités qui lui sont associés (Lucchini, 1993; Parazelli, 1997). Si un tel sentiment d'appartenance a effectivement pu être décelé chez plusieurs des répondants - sentiment se traduisant notamment par l'impression d'avoir trouvé leur place dans le milieu de la rue parmi d'autres jeunes desquels ils se sentent appréciés - ce n'était pas le cas de tous. D'autres répondants, qui venaient de se retrouver à la rue après la perte de leur emploi et de leur appartement, cherchaient au contraire à se distancier à la fois du milieu de la rue et des jeunes qui le fréquentaient et disaient y graviter temporairement dans l'attente d'un nouvel emploi.

C'est dire que les adeptes $\mathrm{du}$ "squeegee» entretiennent des rapports à la rue diversifiés et il en va de même de leur rapport à la pratique ellemême. La place que cette dernière prend dans leur vie varie en fonction de leurs besoins financiers, de leurs objectifs et de leur contexte de vie. D'après les entretiens, le «squeegee» peut être pratiqué temporairement, selon un mode de dépannage, par des jeunes en mauvaise posture financière, notamment après la perte de leur emploi et de leur appartement, dans l'attente d'un nouvel emploi stable; il peut aussi être pratiqué seulement les fins de semaine, sur une base régulière, par des jeunes en appartement et aux études à temps plein, afin de pallier le faible revenu procuré par l'aide sociale et pour pouvoir assumer des responsabilités financières liées à leur appartement et se payer des «extras», comme des cigarettes, des sorties et des drogues douces; il peut enfin occuper une place centrale dans la vie d'autres jeunes, tels ceux qui comptent uniquement sur cette source de revenu ou encore qui doivent entretenir une consommation de drogues injectables relativement coûteuse. Pour eux, la pratique du «squeegee» structure les journées; ils y ont systématiquement recours de façon quotidienne, à raison de plusieurs heures par jour, comme s'il s'agissait d'un travail.

En somme, les adeptes du «squeegee» sont loin de former une catégorie sociale homogène quant à leur rapport à la rue et à la pratique du «squeegee» et quant à leurs objectifs de vie et à leurs besoins financiers; ils ne forment pas, non plus, une catégorie distincte de jeunes parmi l'ensemble des jeunes qui vivent dans les rues montréalaises. Désigner ces jeunes par l'étiquette «squeegee» est une façon de réduire 
leur vie à la pratique qu'ils ont adoptée pour différentes raisons et de leur supposer une expérience de vie univoque alors qu'il n'en est rien. En outre, elle substitue leur identité à leur outil de travail, d'où l'importance de déconstruire cette étiquette. Mais, comme nous l'avons vu, définir les adeptes $\mathrm{du}$ «squeegee» comme des «jeunes de la rue» s'avère également réducteur, puisqu'ils ne développent pas tous un sentiment d'appartenance envers le milieu de la rue. C'est ainsi que la terminologie «jeunes en situation de rue» proposée par Bellot (2001) semble plus appropriée pour définir les adeptes du «squeegee» puisqu'elle a le mérite, d'une part, d'englober la diversité des expériences de vie et des rapports à la rue que peuvent vivre les jeunes qui se retrouvent dans les rues montréalaises et, d'autre part, de ne pas faire de la rue et de la pratique du «squeegee» les aspects le plus importants pour eux. Un adepte du «squeegee» peut donc être vu comme un jeune en situation de rue qui recourt au «squeegee» de façon plus ou moins régulière et systématique pour gagner de l'argent.

\section{De l'acceptation sociale de la pratique du « squeegee » à sa répression}

Le contexte social entourant la pratique du «squeegee» à Montréal s'est transformé au fil des années. Regroupant peu d'adeptes à ses débuts, elle était, d'après les jeunes qui s'y sont initiés en 1994-1995, relativement acceptée par les automobilistes et les policiers. Après avoir gagné en popularité auprès des jeunes en situation de rue, cette pratique est devenue illégale en 1996 (Foisy, 1999), d'abord en vertu d'un règlement municipal ${ }^{5}$, puis par un article du Code de la sécurité routière ${ }^{6}$. Cette décision d'intervenir par la voie répressive aurait été motivée par la volonté de contrer l'expansion massive $\mathrm{du}$ «squeegee» à Montréal, mais aussi par les risques d'accidents qu'il engendrerait en raison de la présence des jeunes directement dans la rue sur les voies de circulation routière. Un autre argument invoqué pour justifier la judiciarisation du «squeegee» touche l'abondance des plaintes d'automobilistes, de citoyens et de commerçants, concernant

5. Il s'agit du règlement municipal C-4, article 21 (B-423) qui interdit de «s'être tenu sur le trottoir, sur la voie publique ou dans un lieu de stationnement dans le but de solliciter la surveillance ou la garde d'un véhicule ou d'offrir ses services pour nettoyer, essuyer ou polir un véhicule, sans permis du directeur».

6. Il s'agit de l'article 448 (P-043) du Code de la sécurité routière qui interdit «d'être sur la chaussée pour traiter avec l'occupant d'un véhicule». 
notamment leur crainte d'être vandalisés, volés ou agressés par ceux qui pratiquent le «squeegee» et, également, leur perception de l'inutilité du service offert (Crago, 1998). C'est ainsi qu'en 1999, au moment de notre étude, le «squeegee» se pratiquait dans un contexte désapprobateur et répressif.

Les adeptes du «squeegee» ont témoigné, lors des entretiens, de relations souvent difficiles, voire tendues, avec les automobilistes et les policiers. En plus de risquer constamment de recevoir des contraventions, ils se sentaient exposés à une désapprobation sociale, c'est-à-dire à divers préjugés sociaux, à une image défavorable d'eux-mêmes, à une étiquette de «drogué», bref, à une stigmatisation sociale et à une non-reconnaissance symbolique de leur valeur d'être humain et de leur utilité sociale. Un tel contexte de désapprobation sociale n'était pas sans conséquence pour eux. Si la plupart disaient essayer, tant bien que mal, de dépasser l'image négative que leur projetaient certains automobilistes, de même que certains de leurs jugements et remarques, ils pouvaient néanmoins en venir à les intérioriser. Une ambivalence à l'égard de la pratique du «squeegee» se dénotait aussi dans leurs récits, surtout en ce qui a trait aux problèmes judiciaires qui l'accompagnaient, voire qu'elle aggravait, en raison des contraventions émises contre eux. Il faut dire que, le plus souvent, les adeptes du «squeegee» ne payaient pas leurs contraventions, que ce soit faute de moyens ou en guise de protestation contre cette mesure judiciaire qu'ils jugeaient absurde. De même, l'émission de contraventions relativement à la pratique $\mathrm{du}$ «squeegee», auxquelles s'ajoutaient des contraventions pour d'autres méfaits ${ }^{7}$, ouvrait la porte à l'emprisonnement ${ }^{8}$. Malgré de telles difficultés sur les plans affectif et judiciaire, les répondants s'estimaient contraints de continuer à pratiquer le «squeegee» pour des raisons financières, quitte à en subir les conséquences. Toutefois, considérant la baisse apparente du nombre d'adeptes du «squeegee» depuis les années 2000 à Montréal, il devient légitime de se demander comment les jeunes en situation de rue qui pratiquaient autrefois le «squeegee» s'y prennent désormais pour gagner de l'argent.

7. Refus de circuler, état d'ivresse sur la voie publique, avoir jeté un mégot de cigarette sur la chaussée et flânage sont autant de méfaits pour lesquels les répondants ont rapporté avoir déjà reçu une contravention.

8. Parmi les quinze répondants, quatre savaient que des mandats d'emprisonnement avaient été émis contre eux, tandis que huit avaient déjà connu une ou plusieurs expériences de détention, entre autres pour des non-paiements d'amendes liées au «squeegee». 


\section{La pratique du " squeegee » comme agent d'une insertion sociale marginalisée}

Malgré les difficultés et l'ambivalence suscitées par un contexte social désapprobateur et répressif, plusieurs motivations amènent les adeptes $\mathrm{du}$ «squeegee» à privilégier la pratique de celui-ci plutôt qu'un autre moyen, au-delà de la possibilité de gains financiers. L'étude du sens de la pratique $\mathrm{du}$ «squeegee» a permis de faire ressortir, notamment, une dimension morale qui lui est associée, de même que la possibilité qu'elle offre d'exprimer une différenciation sociale et de favoriser une socialisation marginalisée. Ces trois éléments de sens invitent à la questionner comme agent d'une insertion sociale marginalisée pour ses adeptes.

Parler d'insertion sociale marginalisée nécessite de relater, au préalable, les circonstances qui ont précédé l'arrivée sur la rue des adeptes du «squeegee» et leur initiation à la pratique. À cet égard, les expériences de vie des répondants rejoignent celles des jeunes de la rue généralement dépeintes dans les études (Comité de travail sur les jeunes sansabri, 1993 ; Fortier et Roy, 1996; Groleau, 1997; Poirier et al., 1999) : histoires familiales le plus souvent ponctuées de conflits, de négligence ou de violence, prises en charge institutionnelles, fugues à répétition du milieu d'origine, difficultés ou conflits scolaires menant au décrochage, expériences de travail limitées, consommation de drogues. En regard de ce type d'expérience de vie, certains auteurs souligneront le processus d'exclusion sociale vécu par les jeunes de la rue (Comité de travail sur les jeunes sans-abri, 1993; Groleau, 1997); d'autres insisteront sur leurs difficultés d'insertion sociale et sur leur inscription dans une trajectoire de vulnérabilité sociale, les amenant à désinvestir les instances de socialisation traditionnelle et à entrer progressivement dans le milieu de la rue (Bellot, 2001). L'expérience de la rue n'est pas sans accentuer leur vulnérabilité sociale et sans les conduire à vivre une forme d'exclusion symbolique (De Gaulejac et Léonetti, 1994); l'écart entre leur mode de vie de rue et le mode de vie «conventionnel», ainsi que la visibilité de leur mode de vie et de certaines de leurs pratiques («squeegee», regroupement dans des parcs, consommation de drogues, etc.), les amènent à se sentir dénigrés et rejetés par la société, étant non conformes aux normes socialement valorisées (Groleau, 1997).

En dépit de la stigmatisation qu'elle peut susciter, l'expérience de la rue peut, selon Parazelli (1997) et Bellot (2001), non seulement représenter pour les jeunes en situation de rue une réponse aux difficultés d'insertion, mais aussi une solution de rechange à une insertion tradi- 
tionnelle. Dès lors, à l'instar de Nicole-Drancourt et Roulleau-Berger (1995) qui stipulent que la précarité d'emploi peut agir, dans certains cas, comme véritable moteur de nouveaux modes d'insertion sociale, nous croyons que la pratique du «squeegee» constitue, pour ses adeptes, l'un des agents d'une insertion sociale autre. Comme nous le montrerons à la lumière des sens qu'elle revêt pour eux, cette pratique semble permettre de s'inscrire de façon non conventionnelle dans les sphères économique, sociale et symbolique de la société, sphères responsables de l'insertion sociale conventionnelle selon De Gaulejac et Léonetti (1994). C'est ainsi qu'ils vivraient une forme d'insertion sociale, quoique marginalisée, puisqu'à l'encontre des normes sociales dominantes.

\subsection{Le «squeegee» comme pratique morale}

La pratique du «squeegee» constitue une activité lucrative qui permet aux jeunes en situation de rue de gagner de l'argent et de pallier un manque de ressources financières. Si le travail conventionnel est le critère par excellence d'une intégration dans la sphère économique de la société en permettant la participation aux activités de production et de consommation (De Gaulejac et Léonetti, 1994), force est de constater qu'il en va de même de la pratique du «squeegee», aussi éloignée soit-elle d'un travail conventionnel. Plus encore, cette pratique revêt même une importante dimension morale pour ses adeptes : elle leur permet de gagner de l'argent d'une manière qui leur semble tout à fait correcte et convenable, aussi bien pour eux-mêmes que pour la société.

Pour bien comprendre cette valeur morale associée à la pratique du «squeegee», il faut nécessairement la comparer avec les autres activités lucratives généralement disponibles pour les jeunes en situation de rue, lesquelles s'avèrent passablement limitées. Hormis la pratique du «squeegee», il s'agit le plus souvent de la sécurité du revenu, de la quête, d'activités criminelles telles la vente de drogues, le vol et la prostitution et, pour certains, d'un travail plus conventionnel (Roy et al., 1996). Le fait d'opter pour un moyen plutôt qu'un autre dépend de la représentation que les jeunes se font de ces différents moyens, de leur contexte de vie et de leur besoin d'argent.

La quête, déjà utilisée par tous les répondants, est perçue par ceux-ci non seulement comme peu rentable mais comme passive. En contrepartie, la pratique du «squeegee» est vue comme un moyen plus dynamique 
de faire de l'argent et leur donne le sentiment de gagner leur argent durement, ce qui les valorise davantage :

Au moins [en faisant du «squeegee»], je suis pas écrasée sur mon cul à rien faire. Je travaille, je fais de quoi pour avoir mon argent. C'est pas comme quêter. J'haïs ça quêter. C'est comme d'attendre que le monde nous fasse vivre à quelque part [...]. En faisant du «squeegee», on dirait que ça me valorise. Je fais de quoi pour l'avoir mon argent (Orélie, 20 ans).

Les activités criminelles (prostitution, vente de drogues, vol) constituent des moyens auxquels certains répondants ont clairement exprimé ne pas vouloir recourir. Pour eux, le «squeegee» parait être l'activité lucrative de rue la plus convenable et la moins risquée en termes de judiciarisation :

Vendre de la drogue, moi je trouve que ça apporte ben des problèmes. Je vois pas pourquoi moi je me ferais pas prendre si les Hell's se font prendre [...]. C'est sûr que vendre de la drogue, c'est plus payant. Mais j'aurais trop peur de me faire prendre. Moi, je fais pas de coups. Je me tiens loin de ça (Jocelyn, 19 ans).

Quant aux adeptes du «squeegee» qui ont déjà été impliqués dans des activités criminelles, leur récit montre que c'est surtout lors d'une consommation importante de drogues, et particulièrement de drogues injectables, qu'ils y ont eu recours. Dans ce cas, les activités criminelles constituent des moyens plus rapides et payants que le «squeegee» pour leur assurer l'entrée d'argent nécessaire à ce type de consommation. Toutefois, leur implication dans de telles activités a entraîné une perception négative d'eux-mêmes, en plus d'une impression de ne pas avoir agi convenablement :

L'été passé, quand je faisais de la poudre, c'est ça que je faisais de la prostitution. Pis je me suis sentie bas en crisse [...]. Faire du «squeegee», dans le fond, je trouve que c'est plus correct. C'est un moyen que je trouve le plus respectable (Natacha, 19 ans).

Avant, je faisais des vols à l'étalage, des piaules aussi. J'ai fait de la prostitution au centre-ville. Faque quand tu sors de thérapie pis que tu vois tout ça, je me sentais mal d'avoir fait tout ça. C'est pour ça que je me suis dit que j'allais squeeger. Au moins, je vais me sentir mieux avec moi-même pis je me disais que $j$ 'allais pas retomber dans toute cette patente-là de me geler pis d'aller faire des passes après (Thomas, 26 ans).

Pour ce qui est du travail conventionnel, il faut dire qu'au moment des entretiens, plusieurs répondants le rejetaient catégoriquement. Pour certains d'entre eux, il s'avère le symbole parfait d'une adhésion au système 
capitaliste et aux valeurs d'argent et de pouvoir qui lui sont associées et qu'ils désapprouvent; pour d'autres, notamment ceux qui poursuivent des études à temps plein tout en recevant des prestations de la sécurité du revenu, avoir un emploi tout en étudiant équivalait à leurs yeux à une détérioration de leurs conditions de vie actuelles vu la baisse de revenu qui s'ensuivrait. Et pour les répondants qui aspirent précisément à obtenir un emploi stable, le fait de ne pas avoir d'appartement et une scolarisation limitée entravaient considérablement cette possibilité.

Bref, on constate que non seulement la pratique du «squeegee» permet à ses adeptes de demeurer ancrés dans la sphère économique de la société en leur fournissant les ressources financières nécessaires sinon pour vivre, du moins pour survivre, mais qu'elle est perçue par eux comme étant l'un des seuls moyens disponibles dans le milieu de la rue pour gagner de l'argent de façon convenable, sans contrevenir à leur intégrité, à leurs valeurs et à leur estime de soi.

\subsection{Le «squeegee» comme pratique de différenciation sociale}

La pratique du «squeegee» permet aussi à ses adeptes d'exprimer un désir de différenciation sociale. Plusieurs d'entre eux souhaitent se dissocier, temporairement ou plus définitivement, d'un mode de vie conventionnel par l'adoption d'un mode de vie de rue perçu comme différent et pouvant leur permettre de vivre leur vie selon leurs propres désirs. $\mathrm{Si}$ la vie conventionnelle correspond pour eux à la stabilité, à la routine, aux responsabilités et à l'occupation d'un emploi stable, le mode de vie de rue prend plutôt la forme d'une quête de vitalité et de liberté :

Je me revois quand $j$ 'avais mon appart pis ma petite routine. Je l'ai eu ce beat là pendant 2 ans. À quelque part, j'étais pas bien parce qu'il me manquait quelque chose. Genre de me casser la tête pour pas me lever en retard pis pour pas arriver à ma job en retard pis de prévoir ma vie, ben moi, j'aimais pas ça. Là, c'est vraiment je passe par là pis j'ai le goût de faire ça pis c'est ça que je fais. C'est la sensation de vivre vraiment, c'est ça que j'aime (Orélie, 20 ans).

La vie de rue se traduit pour ses adeptes non seulement par une absence d'obligations, de contraintes et de responsabilités, mais aussi par la possibilité de faire leurs propres choix, de décider par eux-mêmes et pour eux-mêmes, en sentant qu'aucune contrainte extérieure ne peut contrecarrer leurs projets et leurs désirs :

J'aime ça être libre. La routine, ça m'énerve. [...]. Pour moi, c'est plus intéressant d'avoir des amis pis d'être libre que d'avoir de l'argent pis tous les pro- 
blèmes qui viennent avec. C'est du cassage de tête pour rien tout ça. Là, je suis quand même assez libre, j'ai pas d'attaches [...]. J'ai rien qui me retient pis je peux aller où je veux. J'ai pas de «il faut que je revienne payer mon loyer». Je peux partir 3, 4, 6 mois ou 1 an si ça me tente, il y a personne qui va chialer. Dans le fond, j'ai pas de responsabilités ici, faque je peux partir pour squeeger ailleurs (Hubert, 20 ans).

Le mode de vie de rue et la pratique du «squeegee» permettent ainsi à des adeptes de mener leur vie selon leurs désirs, d'une manière différente, mais plus complète et cohérente à leurs yeux, selon des principes qui leur semblent incompatibles avec un mode de vie conventionnel. Bien que, d'un point de vue social, la pratique du «squeegee» est vue comme une activité en marge de la société conventionnelle, il faut voir que certains adeptes cherchent précisément à se dégager de cette société à laquelle correspondent selon eux des normes, des principes et un mode de vie qui ne répondent ni à leurs besoins, ni à leurs désirs immédiats.

\subsection{Le «squeegee» comme pratique de socialisation marginalisée}

La pratique du «squeegee» posée comme «pratique de socialisation marginalisée», selon la terminologie de Parazelli (1997), a la particularité de favoriser, chez des jeunes qui l'ont adoptée, le développement d'un sentiment d'appartenance au milieu de la rue, tout en étant marginalisée du point de vue des normes sociales en place.

Un fort désir de socialisation ressort du récit de plusieurs répondants, c'est-à-dire un désir de se sentir aimé, reconnu et d'appartenir à un groupe, et c'est le milieu de la rue qui leur offre la possibilité de le combler. Ils ont le sentiment d'y trouver leur place parmi d'autres jeunes en situation de rue avec lesquels ils partagent un mode de vie et des valeurs similaires :

Je voulais tout le temps m'en aller [...] au centre-ville pis dans les ressources. Il y a gros du monde ici pis au moins ils t'apprécient [...]. Moi, quand j'allais au centre-ville, je me sentais bien. Je voyais les autres jeunes pis je leur parlais. J'étais bien (Nathaniel, 20 ans).

La pratique du «squeegee» permet ainsi de marquer leur adhésion au milieu de la rue et de favoriser la création de liens significatifs avec d'autres jeunes du milieu. Soulignons l'existence dans le milieu de la rue de règles de conduite tacites régissant les relations sociales et les activités, dont la pratique du «squeegee», auxquelles des répondants ont 
affirmé devoir souscrire, du moins à l'intérieur de leur groupe d'amis respectif. Celles-ci concernent généralement le respect, l'entraide et la solidarité :

Des fois, on squeege pis il y en a qui font moins de cash que d'autres. Faque on se met toute ensemble pis on se paye des affaires à la gang ou on lui paye ce qu'il a besoin. On fait souvent des échanges de même [...]. On s'aide.

C'est vraiment la solidarité. C'est une grande famille (Orélie, 20 ans).

La solidarité se traduit également par des codes verbaux ou non verbaux servant à s'avertir entre eux de l'arrivée au loin des policiers.

La pratique du «squeegee» semble donc permettre à certains de ses adeptes la création de liens sociaux significatifs avec d'autres jeunes en situation de rue avec lesquels ils partagent certaines affinités et valeurs. Cette pratique devient ainsi pour eux un agent d'intégration au milieu de la rue et leur permet d'y vivre selon les règles de conduite en vigueur et de consolider leur sentiment d'appartenance envers le milieu de la rue et, surtout, envers leur groupe d'amis respectif. Par ailleurs, il est important de signaler que plusieurs répondants fréquentaient certaines institutions sociales telles l'école, de même que les organismes communautaires qui offrent des services adaptés à leurs besoins. Les adeptes du "squeegee», comme la plupart des jeunes en situation de rue, sont plus enclins à consulter des intervenants oeuvrant dans de tels organismes, car ils s'y sentent reçus sans jugement et peuvent y trouver soutien et disponibilité, contrairement aux attitudes auxquelles ils craignent d'être confrontés dans les services sociaux et dans les services de santé officiels (Parazelli, 1996). C'est dire que les adeptes du «squeegee» demeurent ancrés de façon marginalisée dans le social, à travers les individus et les ressources propres au milieu de la rue.

D'un point de vue symbolique, il est vrai que le mode de vie des adeptes $\mathrm{du}$ «squeegee» s'éloigne du mode de vie conventionnel et que la visibilité de leur mode de vie de même que celle de la pratique du «squeegee» peut les amener autant à vivre des relations difficiles avec certains automobilistes et policiers qu'à se sentir stigmatisés et rejetés par la société. Mais, en revanche, la pratique du «squeegee» en amène plus d'un à trouver sa place au sein du milieu de la rue et d'un groupe d'amis, à y développer un sentiment d'appartenance et à y acquérir un statut, aussi marginalisé soit-il.

Il faut cependant insister sur le fait que la pratique du «squeegee» n'est pas toujours liée à un mode de vie de rue et au développement d'un sentiment d'appartenance envers le milieu de la rue puisque, comme 
nous l'avons vu précédemment, certains adeptes du «squeegee» désiraient une vie plus conventionnelle. Bien que, pour ces derniers, la pratique $\mathrm{du}$ «squeegee» ne constitue pas une pratique de différenciation sociale, ni même de socialisation marginalisée, leur entrée temporaire dans le milieu de la rue, et leur investissement tout aussi temporaire dans la pratique du «squeegee», leur permettent néanmoins de bénéficier des ressources et des opportunités offertes par ce milieu, comme le montrent les propos de Jocelyn relatant son arrivée à Montréal un an avant notre entretien :

Quand je suis arrivé, j'étais dans la rue. J'avais entendu parler des organismes pis je voulais savoir c'était quoi. Je suis venu dîner [dans un organisme] pis j'ai rencontré un gars que je suis allé habiter chez eux avec ses colocs. Punk, c'est pas mon style, mais j'ai rien contre les punks. C'est un style de vie, une mentalité. Je m'habillerais pas comme eux, mais j'ai rien contre ça. J'ai appris à les connaître pis à vivre avec eux. Je suis resté chez eux 2-3 semaines, j'ai fait du «squeegee» pis j'ai cherché un emploi. Après, je suis allé en appart pis je me suis débrouillé (Jocelyn, 19 ans).

Lors de l'entretien, Jocelyn vivait toujours une situation de rue, c'est-àdire qu'il était sans domicile, fréquentait les ressources communautaires et pratiquait le «squeegee» dans l'attente d'un nouvel appartement et d'un nouvel emploi. Son expérience, tout comme celle d'autres répondants qui désiraient une vie plus conventionnelle, laisse à penser que le milieu de la rue, sans être entièrement investi, constituerait un milieu social leur servant de levier pour l'atteinte de leurs objectifs de vie à court terme. Quoi qu'il arrive, ils savent que ce milieu leur est ouvert, qu'ils pourront y trouver du soutien, tant de la part des ressources communautaires que des jeunes qui s'y trouvent, et qu'ils pourront pratiquer le «squeegee» pour gagner un peu d'argent.

En somme, à partir des différents sens que prend la pratique du «squeegee» pour ses adeptes, c'est-à-dire une pratique morale, une pratique de différenciation sociale et une pratique de socialisation marginalisée, il est possible de croire qu'elle permet, par ses dimensions lucrative, socialisante et symbolique, de les ancrer dans une forme d'insertion sociale non conventionnelle. Car, au bout du compte, une non-insertion à la société conventionnelle et à ses institutions n'implique pas nécessairement une exclusion de la société, ce que les études sur les jeunes de la rue avaient tendance à conclure dans les années 1990. Lorsque Barel (1989) souligne que le système social se définit à la fois par les éléments qui se forment en lui et par les éléments qui se forment à côté de lui, il met précisément en lumière le 
fait que les marginaux, les hors-normes, font inévitablement partie du système social. Et comme le mentionne Parazelli (2002 : 326), la marginalisation sociale «n'expulse pas la personne du social, mais l'y ancre à sa périphérie». C'est pourquoi l'expérience des jeunes adeptes du «squeegee» rencontrés donne à penser à une insertion sociale marginalisée qui, en plus de favoriser le développement d'un réseau de solidarité, leur procure une existence au sein d'un monde social qui peut leur convenir d'une manière temporaire ou plus prolongée et qui les accepte.

\section{Conclusion}

Derrière la pratique du «squeegee» se cache une multiplicité d'expériences et de modes de vie. Non seulement ses adeptes entretiennent-ils différents rapports au milieu de la rue et à la pratique elle-même, mais l'argent qu'elle leur permet de gagner sert à de nombreuses fins, depuis le loyer, l'achat de nourriture, de vêtements et de drogues douces, jusqu'au soutien d'une consommation de drogue injectable. L'étiquette par laquelle ils sont généralement désignés ne rend toutefois pas compte de la diversité de leurs expériences de vie, d'où l'importance de la déconstruire. Tout compte fait, le terme «squeegee» ne nomme qu'une activité à laquelle des jeunes en situation de rue peuvent recourir pour gagner de l'argent.

Malgré un contexte social désapprobateur et répressif, en 1999 du moins, et des relations plutôt difficiles avec certains automobilistes et policiers, les adeptes $\mathrm{du}$ «squeegee» perçoivent leur pratique comme une activité morale qui leur permet de gagner de l'argent en préservant leur estime de soi et en causant le moins de préjudice possible à eux-mêmes comme à autrui. Elle apparaît aussi pour certains comme une façon d'exprimer un désir de différenciation sociale et de s'inscrire dans un mode de vie différent basé sur des principes de liberté, de vitalité et de spontanéité qui leur semblent incompatibles avec un mode de vie conventionnel. Cette activité semble, enfin, favoriser cette «socialisation marginalisée» dont parle Parazelli, puisqu'elle agit, pour plusieurs adeptes, comme un agent d'intégration au milieu de la rue et leur permet d'y trouver une place parmi d'autres, desquels ils se sentent reconnus et appréciés. Par ses dimensions lucrative, sociale et symbolique, cette pratique favoriserait une insertion sociale marginalisée. Sans nier les difficultés auxquelles les adeptes du «squeegee» peuvent être confrontés, le fait de concevoir ainsi la pratique de cette activité met en évidence leur désir d'existence sociale. 


\section{Références}

Barel, Y. (1989). Le paradoxe et le système : essai sur le fantastique social. Grenoble : Presses Universitaires de Grenoble.

Bellot, C. (2001). Le monde social de la rue : expériences des jeunes et pratiques d'intervention à Montréal. Thèse de doctorat, École de criminologie, Université de Montréal.

Brannigan, A., \& Caputo, T. (1993). Études sur les jeunes fugueurs et les jeunes de la rue au Canada : problèmes conceptuels et méthodologiques. Ottawa : Solliciteur Général du Canada.

Comité de travail sur les jeunes sans-abri. (1993). Réflexion sur la situation des jeunes sans-abri. Rapport du comité de travail à l'attention du Comité de suivi du Programme conjoint Ville de Montréal - MSSS.

Courville, V. (1998). La pratique du squeegeeing chez les jeunes marginaux. Service de police de la Communauté urbaine de Montréal, division Planification et orientations stratégiques.

Crago, A.-L. (1998). Squeegee. Rapport inédit.

De Gaulejac, V., \& Léonetti, I. (1994). La lutte des places. Paris : Desclée de Brouwer.

Foisy, C. (1999). Le squeegeeing au centre-ville de Montréal : perceptions et réalité. Rapport conjoint Ville de Montréal/Spectre de Rue.

Fortier, J., \& Roy, S. (1996). Les jeunes de la rue et l'intervention : quelques repères théoriques. Cabiers de recherche sociologique, 27, 127-152.

Grenier, E. (1997). Revue de l'année 1997. Voir, 23 décembre, p. 8.

Groleau, L. (1997). Exclusion et identité : trajectoires d'exclusion, construction identitaire et santé mentale des jeunes sans-abri. Intervention, 105, 55-67.

Laberge, D., \& Roy, S. (1994). Marginalité et exclusion sociales : des lieux et des formes. Cabiers de recherche sociologique, 22, 5-9.

Lucchini, R. (1993). Enfant de la rue : identité, sociabilité, drogue. Genève : Librairie Droz.

Nicole-Drancourt, C., \& Roulleau-Berger, L. (1995). L'insertion des jeunes en France. Paris : Presses Universitaires de France.

Parazelli, M. (1996). Les pratiques de socialisation marginalisées des jeunes de la rue dans l'espace urbain montréalais. Cabiers de recherche sociologique, 27, 47-61.

Parazelli, M. (1997). Pratiques de «socialisation marginalisée» et espace urbain : le cas des jeunes de la rue à Montréal (1985-1995). Thèse de doctorat, Département d'études urbaines, Université du Québec à Montréal.

Parazelli, M. (2002). La rue attractive : parcours et pratiques identitaires des jeunes de la rue. Québec : Presses de l'Université du Québec.

Pigeon, M. (1999). Voyage en enfer. Le journal de Montréal, 19 septembre, p. 3.

Poirier, M., Lussier, V., Letendre, R., Michaud, P., \& Morval, M. (1999). Relations et représentations interpersonnelles de jeunes adultes itinérants. Montréal : GRIJA. 
Poupart, J. (1997). L'entretien de type qualitatif : considérations épistémologiques, théoriques et méthodologiques. In J.-P. Deslauriers, L.-H. Groulx, A. Laperrière, R. Mayer \& J. Poupart (eds), La recherche qualitative : enjeux épistémologiques et méthodologiques (173-209). Boucherville: Gaétan Morin.

Roy, É., Haley, N., Boivin, J.-F., Frappier, J.-Y., \& Claessens, C. (1996). Les jeunes de la rue de Montréal et l'infection au VIH. Montréal : Unité de Santé publique. 